\section{Continuous high dosing of lenalidomide in relapsed, refractory or older newly diagnosed acute myeloid leukemia patients not suitable for other treatment options - results from a phase I study}

Lenalidomide is an immunomodulatory drug approved for multiple myeloma, relapsed mantle cell lymphoma and myelodysplastic syndrome (MDS) with deletion of chromosome $5 \mathrm{q}(\operatorname{del}(5 \mathrm{q}))$. Based on the high rate of hematologic improvement and cytogenetic responses seen in $\operatorname{del}(5 q)$ MDS, several trials have been conducted with lenalidomide in acute myeloid leukemia (AML) and highrisk MDS irrespective of the presence of $\operatorname{del}(5 q)$. A response rate of $30 \%$ in a high dosing schedule was reported in older newly diagnosed AML patients. ${ }^{1}$ In contrast, in a phase I trial with refractory and relapsed $(\mathrm{r} / \mathrm{r}-)$ AML patients, the response rate was lower and a high toxicity was observed when lenalidomide was given at higher doses of up to $75 \mathrm{mg} /$ day over a 21-day time period. ${ }^{2}$

Based on the initial encouraging reports, ${ }^{3,4}$ we performed a phase I dose escalating ( $3+3$ design) study with single agent continuous lenalidomide. Patients with either relapsed/refractory AML or older patients with newly diagnosed AML (age $>60$ years) unfit for intensive treatment were eligible. clinicaltrials. gov identifier 00839059.

Standard in- and exclusion criteria for phase I doseescalation trials were used including the absence of polyneuropathy at enrolment; out-patient treatment was intended. The starting dose was $25 \mathrm{mg}$ lenalidomide/day as continuous treatment until complete remission (CR), dose-limiting toxicity (DLT) or day 56.

Between March 2009 and June 2010, 14 patients (median age 71.5 years, range $35-83$ years) were enrolled and 12 patients (1 screening failure, 1 rapidly progressive disease) were treated in 2 dose levels ( $25 \mathrm{mg}, 50 \mathrm{mg}$ ). Eight patients were male, 8 patients had r/r-AML, and 4 patients had newly diagnosed AML. Median number of prior lines of therapies was 1 (range $0-5), 8$ patients $(67 \%)$ had adverse cytogenetics including 5 cases with del(5q) and 4 had a normal karyotype; median white blood cell count at study entry was $3.5 \mathrm{G} / 1$ (range 0.7-27.3 G/1).

Five patients were treated at a dosage of $25 \mathrm{mg} /$ day without DLT and, subsequently, 7 patients at a dosage of $50 \mathrm{mg} /$ day. At a dosage of $50 \mathrm{mg} /$ day, 2 DLTs were observed with polyneuropathy CTC AE grade III. This is in contrast to other phase I trials with lenalidomide in AML where haematological toxicities or fatigue were dose limiting. No cytokine release syndrome was seen within the trial, but 2 patients developed drug hypersensitivity reaction CTC AE grade III with eyelid edema.

Only 7 patients $(58 \%)$ were eligible for first response evaluation at day 22; the other 5 patients dropped out before due to rapid progressive disease $(n=3)$ or severe infectious complications ( $n=2$; urosepsis and pneumonia). None of the patients achieved a PR or CR. In 1 patient, a decrease in bone marrow blasts from $40 \%$ to $15 \%$ and blast clearance in peripheral blood was documented; treatment was discontinued at day 47 based on worsening of anemia and thrombocytopenia to CTC grade 4 and heavily impaired quality of life. Overall, treatment discontinuation was due to progressive disease $(n=7)$, DLT $(n=2)$, worsening of general condition $(n=1)$, patient's wish $(n=1)$ and death $(n=1)$. Ten patients were hospitalized during treatment due to serious adverse events not manageable in an outpatient setting. The 30-day and 60day mortality was $42 \%(5 / 12)$ and $50 \%(6 / 12)$, respective- ly. At day 140 after study entry, the last patient had died.

Our results are in line with the data from Zeidan et al., showing poor feasibility and outcome of higher dosages of lenalidomide in AML. ${ }^{5}$ DLT in our study was polyneuropathy CTC AE grade III, which has not been observed in other studies evaluating higher dosages of lenalidomide.

A combination therapy of low-dose lenalidomide with low- or intermediate-dose cytarabine showed feasibility but only minimally superior results compared to single agent treatment with cytarabine in two phase-I/II single arm trials. ${ }^{6,7}$

Recently, the combination treatment of a hypomethylating agent and lenalidomide was reported in AML. ${ }^{8}$ In a randomized 3-arm study, lenalidomide was administered at a high dosage as monotherapy $(50 \mathrm{mg} /$ day for 28 days in the first 2 cycles) or sequentially to azacytidine. Again, poor tolerability and low efficacy was reported in this elderly population with newly diagnosed AML.

The high sensitivity of $\operatorname{del}(5 q)$ MDS to lenalidomide results from haploinsufficiency of casein kinase 1A1 (CSNK1A1) and degradation of this protein induces apoptosis by activating TP53. ${ }^{9}$ Therefore, a prerequisite for the specific activity of lenalidomide in myeloid malignancies is a heterozygous deletion on chromosome 5 comprising the CSNK1A1 gene as well as an intact TP53 pathway. However, in contrast to MDS, deletion of chromosome 5 in AML is highly associated with TP53 mutations, providing a mechanistic explanation as to why lenalidomide has no activity in AML.

In conclusion, our results add to previous knowledge that high dose lenalidomide in relapsed and refractory as well as elderly treatment naïve AML is not feasible and has limited efficacy as single agent therapy.

Marie Luise Hütter-Krönke, ${ }^{1}$ Walter Fiedler, ${ }^{2}$ Andrea Kündgen, ${ }^{3}$ Jürgen Krauter, ${ }^{4,7}$ Marie von LilienfeldToal, ${ }^{5,8}$ Hartmut Döhner ${ }^{1}$ and Richard F. Schlenk ${ }^{1,6}$

'Department of Internal Medicine III, University of Ulm; ${ }^{2}$ Department of Internal Medicine II, University Hospital Hamburg-Eppendorf, Hamburg; ${ }^{3}$ Department of Hematology, Oncology and Clinical Immunology, Heinrich-Heine University Düsseldorf; ${ }^{4}$ Department of Internal Medicine III, Hospital Braunschweig; ${ }^{5}$ Department of Internal Medicine II, University Hospital Jena; ${ }^{6}$ National Center for Tumor Diseases, Heidelberg; ${ }^{7}$ Department of Hematology, Hemostasis, Oncology and Stem Cell Transplantation, Hannover Medical School and Internal Medicine III, University Hospital of Bonn, Germany

Correspondence:marie-luise.huetter@uniklinik-ulm.de doi:10.3324/haematol.2018.199794

Acknowledgments: we thank Celgene for supporting the AMLSG 08-07 trial with the study drug and the members of the German-Austrian AML study group (AMLSG) for including patients. We also thank the members of the study team at Ulm University Martin Bommer, Konstanze Döhner, Verena Gaidzik, Jochen Greiner, Ute Gruesshaber, Silja Mack, Frank Rücker, Stephan Stilgenbauer Andreas Viardot and Daniela Weber for coordination, documentation of the trial and including patients.

Information on authorship, contributions, and financial \& other disclosures was provided by the authors and is available with the online version of this article at www. haematologica.org.

\section{References}

1. Fehniger TA, Uy GL, Trinkaus $\mathrm{K}$, et al. A phase 2 study of highdose lenalidomide as initial therapy for older patients with acute myeloid leukemia. Blood. 2011;117(6):1828-1833

2. Blum W, Klisovic RB, Becker $\mathrm{H}$, et al. Dose escalation of lenalido- 
mide in relapsed or refractory acute leukemias. J Clin Oncol. 2010;28(33):4919-4925.

3. Mesa RA, Tefferi A, Li CY, Steensma DP. Hematologic and cytogenetic response to lenalidomide monotherapy in acute myeloid leukemia arising from JAK2(V617F) positive, del(5)(q13q33) myelodysplastic syndrome. Leukemia. 2006;20(11):2063-2064

4. Fehniger TA, Byrd JC, Marcucci G, et al. Single agent lenalidomide induces complete remission of acute myeloid leukemia in patients with isolated trisomy 13. Blood. 2009;113(5):1002-1005.

5. Zeidan AM, Smith BD, Carraway HE, Gojo I, DeZern A, Gore $\mathrm{SD}$. A phase 2 trial of high dose lenalidomide in patients with relapsed/refractory higher-risk myelodysplastic syndromes and acute myeloid leukaemia with trilineage dysplasia. Br J Haematol. 2017; 176(2):241-247.

6. Visani G, Ferrara F,Di Raimondo F, et al. A Low-dose lenalidomide plus cytarabine in very elderly, unfit acute myeloid leukemia patients: Final result of a phase II study. Leuk Res. 2017;62:77-83.

7. Griffiths EA, Brady WE, Tan W, et al. A phase I study of intermediate dose cytarabine in combination with lenalidomide in relapsed/refractory acute myeloid leukemia. Leuk Res. 2016;43:44-48

8. Medeiros BC, McCaul K, Kambhampati S, et al. Randomized study of continuous high-dose lenalidomide, sequential azacitidine and lenalidomide or azacitidine in persons 65 years and over with newly-diagnosed acute myeloid leukemia. Haematologica. 2018;103(1):101-106.

9. Krönke J, Fink EC, Hollenbach PW, et al. Lenalidomide induces ubiquitination and degradation of CK1 $\alpha$ in $\operatorname{del}(5 q)$ MDS. Nature. 2015; 523(7559):183-188 